\title{
ON SCALE DEFINITION WITHIN CALIBRATION OF MULTI-CAMERA SYSTEMS IN MULTIMEDIA PHOTOGRAMMETRY
}

\author{
O.Kahmen ${ }^{1 *}$, R.Rofallski ${ }^{1}$, N.Conen ${ }^{1}$, T.Luhmann ${ }^{1}$ \\ ${ }^{1}$ Jade University of Applied Sciences, Institute for Applied Photogrammetry and Geoinformatics (IAPG), Ofener Str. 16/19, 26121 \\ Oldenburg, Germany - (Oliver.Kahmen, Robin.Rofallski, Niklas.Conen, Thomas.Luhmann)@jade-hs.de
}

\section{Commission II - WGII/9}

KEY WORDS: Underwater photogrammetry, multi-camera calibration, scale definition, relative orientation, stereo photogrammetry, simulated data

\begin{abstract}
:
In multimedia photogrammetry, multi-camera systems often provide scale by a calibrated relative orientation. Camera calibration via bundle adjustment is a well-established standard procedure in single-medium photogrammetry. When using standard software and applying the collinearity equations in multimedia photogrammetry, the refractive interfaces are modelled in an implicit form. This contribution analyses different calibration strategies for bundle-invariant interfaces. To evaluate the effects of implicitly modelling the refractive effects within a bundle adjustment, synthetic datasets are simulated. Contrary to many publications, systematic effects of the exterior orientations can be verified with simulated data. The behaviour of interior, exterior and relative orientation parameters is analysed using error-free synthetic datasets. The relative orientation of a stereo camera shows systematic effects, when the angle of convergence varies and when the synthetic interface is set up at different distances to the camera. It becomes clear, that in most cases the implicit modelling is not suitable for multimedia photogrammetry. An explicit modelling of the refractive interfaces is implemented into a bundle adjustment. This strict model is analysed and compared with the implicit form regarding systematic effects in orientation parameters as well as errors in object space. In a real experiment, the discrepancies between the implicit form using standard software and the explicit modelling using our own implementation are quantified. It is highly advisable to model the interfaces strictly, since the implicit modelling might lead to relevant errors in object space.
\end{abstract}

\section{INTRODUCTION}

Photogrammetry dealing with more than one optical medium is known as multimedia photogrammetry. It becomes increasingly important in underwater applications such as fish farming, industrial inspection tasks or surveying of archaeological and cultural heritage sites. Camera calibration via bundle adjustment is a well-established standard procedure in single-medium photogrammetry and might also be a reasonable tool for multimedia photogrammetry. In case of underwater photogrammetry, the waterproof camera housings and the water itself bend the optical path of light. These refractive effects need to be compensated implicitly by the standard calibration parameters or modelled explicitly by an extended camera model within the calibration process. Especially non-experts often use standard software to determine calibration parameters of single or multi-camera systems, which usually does not model an extended camera model explicitly. In some cases, depending on the calibration strategy and on the photogrammetric network, neglecting the explicit modelling of refractive effects might cause systematic errors. In case no absolute error in object space is quantified, these effects are not necessarily visible to the user and might remain undetected. We hypothesize that, when scale is provided only by the relative orientation of a multi-camera system, systematic errors occur in forward intersection due to the inevitable correlations between the interior and exterior orientation of a multi-camera system resulting in incorrect scaling.

\section{CALIBRATION TECHNIQUES IN MULTIMEDIA PHOTOGRAMMETRY}

\subsection{Planar interfaces}

In the field of underwater photogrammetry, two main approaches of underwater camera calibration exist regarding planar interfaces. A comprehensive overview of calibration techniques is given by (Shortis, 2015). In general, homogeneous and isotropic interfaces are assumed. The first approach compensates refraction effects implicitly within the standard pinhole camera model. This allows for the usage of standard photogrammetric software to calibrate the camera in a selfcalibration process. The second approach models the refraction effects caused by interfaces explicitly via raytracing (e.g. Kotowski, 1987). Mostly the parameters of the interfaces (orientation, thickness and refractive indices) are assumed to be known (e.g. Bräuer-Burchardt et al., 2015). Under the assumption that one plane of a local coordinate system is parallel to the plane interface, the simplified approach of (Maas, 1992) can be used. (Maas, 2015) proposed that his modular geometric model can easily be integrated in standard photogrammetric software. In this approach a radial shift of an underwater object point with respect to the camera nadir is computed, thus the collinearity condition is fulfilled. Furthermore, it allows for the introduction of the refractive indices into the bundle adjustment as unknowns. However, the author addresses the instability of the mathematical system when introducing refractive indices as unknowns, especially when introducing more than one index. The more complex strict approach by (Kotowski, 1987) generically models the number,

\footnotetext{
* Corresponding author
} 
shape and orientation of interfaces within the adjustment process without any restrictions regarding the orientation of the interfaces. All interface parameters can be solved via the bundle adjustment as implemented in a flexible approach by (Mulsow, 2010).

\subsection{Hemispherical interfaces}

Besides planar interfaces, hemispherical interfaces or dome ports can be mounted on a camera as an interface of the underwater housing. When the projection centre is mounted in the centre of the hemispheric port, this kind of port does not refract the ray of light, since every ray to the centre of projection hits the surface of the dome port perpendicularly. It keeps the field of view as it is in air, thus the standard photogrammetric model can be applied. (Menna et al., 2017a) compare flat and dome ports in an experimental work. The calibration of dome ports as well as the robustness towards an imperfect setup are investigated by (Nocerino et al., 2016). The advantages of dome ports compared to flat ports regarding the geometrical and optical characterisation are discussed in (Menna et al., 2016) and (Menna et al., 2017b).

\subsection{System configuration and calibration strategies}

Several contributions discuss calibration techniques, however, the exterior orientation is rarely considered. (Shortis et al., 2007) provide a number of case studies for underwater stereo systems. The relative orientation can be calibrated within the bundle adjustment if it is introduced as a constraint. Using stereo photogrammetry, the scale may be provided by either a reference object, so that the exterior orientations can be determined simultaneously, or by the pre-calibrated relative orientation of the stereo system itself. According to (Menna et al., 2013) using a stereo system, additional equipment for the scale definition problem becomes obsolete, which is stated as an advantage for underwater inspection utilized by a ROV. From our point of view, this statement only holds true as long as the refractive interfaces are modelled explicitly. Using planar interfaces and the implicit estimation of interior and relative orientation, the bundle adjustment leads to significant errors when scale is provided by the relative orientation only. Depending on the camera configuration and the distance to the object, these errors can be larger or smaller. Furthermore, using the relative orientation for scale definition in navigation tasks might lead to significant errors when multi-camera systems are used such as in (Rofallski and Luhmann, 2018).

Figure 1 illustrates some conceivable setups of different photogrammetric multimedia systems and their calibration approaches. In the first two rows one element becomes part of the physical system. Depending on the number of cameras different approaches are possible. While bundle adjustment approaches 1 and 2 (single and multi-camera) model the refraction effects implicitly, adjust only the interior orientation (IOR) and, if applicable, the relative orientation (ROR), approaches 3, 4 and 5 model the interfaces (IF) explicitly. Strategies 1 and 2 can be applied by using standard software, however, to determine the interior orientation in air (marked red) would automatically lead to gross scale errors for which reason this solution is not considered any further. Approaches 2 are performed in many cases using standard software. (Drap et al., 2015) uses the relative orientation to provide scale and the interior orientation is assumed to be known by pre calibration. Approach 3 is investigated for a single camera bundle (objectinvariant) by (Mulsow, 2010) but, best to our knowledge, not for a multi-camera system yet. The green marked approaches 4 and 5 are investigated for multi-camera systems in this work exclusively for bundle-invariant interfaces, showing that approach 2 does lead to significant deviations in most cases. The approaches 4 and 5 model the interfaces explicitly, thus probably not leading to a scale error in case of multi-camera system usage. Approach 5 gives the possibility to rearrange the cameras and recalibrate the relative orientation, with cameras still mounted in their housing. In contrast, approach 4 calibrates the relative orientation in air, thus the cameras need to be separated from their housings when their arrangement is changed. From a practical point of view this approach is marginal realistic, since the interfaces need to be mounted after the first calibration of the interior and relative orientation without alternating their already calibrated parameters. Thus, in Figure 1 "Air" means no interface being present in front of a camera.

The following section will discuss the case of single and multicamera systems using planar interface (bundle-invariant) in an implicit form.

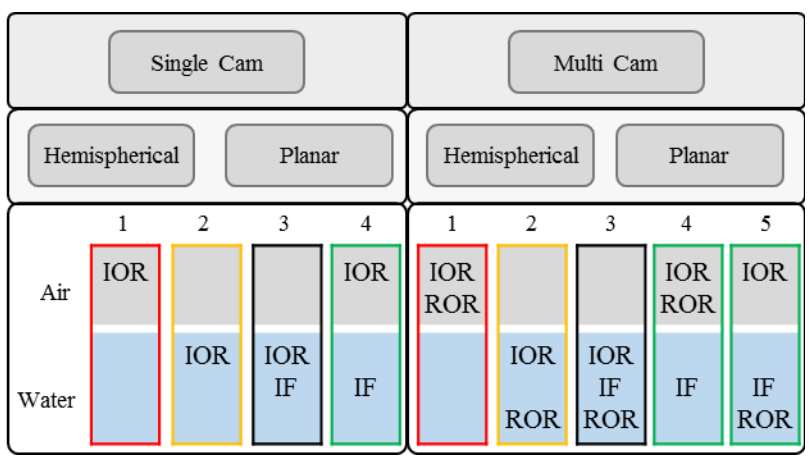

Figure 1. System components, calibration approaches for photogrammetric underwater systems. Left side illustrates options of configuration for single camera usage; right side illustrates options for multi-camera systems. Red frames indicate unreasonable strategies, yellow frames indicate standard strategies using standard software, black frame strategies are not part of this work but theoretically possible, green frames indicate explicit modelling of refractive effects using a known IOR.

\section{ANALYSIS OF CALIBRATION AND ORIENTATION FOR PLANAR INTERFACES IN IMPLICIT FORM}

In this section the analysis of the interior, exterior and relative orientation parameters for single and multi-camera systems is based on synthetic data. By using exterior orientations of a photogrammetric bundle and a fixed interior orientation exclusive of distortions, any object point can be projected into the image via the collinearity equations. Thus, error-free image coordinates unaffected by degradation or noise are determined. By integrating a strict raytracing model using synthetic interfaces, the object points can also be projected into the image through refracting interfaces. All following data in this section refer to the dataset used for synthetic analysis. The synthetic dataset of the photogrammetric bundle consists of 100 object points and 36 well distributed exterior orientations. It is based on an accuracy evaluation test according to the German guideline VDI/VDE 2634.1 (see Figure 2). 


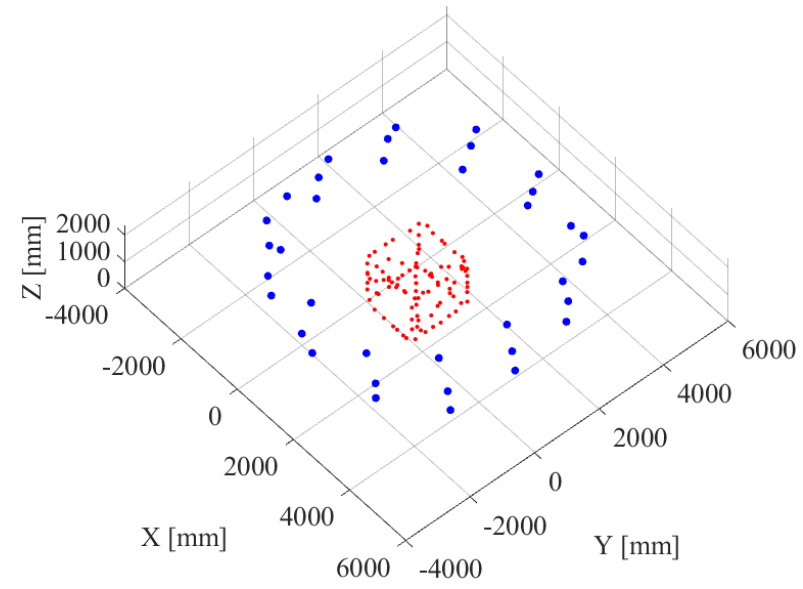

Figure 2. Setup of the synthetic dataset. Blue points indicate the positions of the camera, red points indicate the cubic arranged object points.

The simulated refraction occurs at the plane interfaces perpendicular to the optical axes, air/glass and glass/water (see Figure 3). The refractive indices used in backward ray tracing, according to (Kotowski, 1987) and integrated similar to (Mulsow, 2010), to determine image coordinates are set as:

$\mathrm{n}_{\text {Air }}=1.00$

$\mathrm{n}_{\text {Glass }}=1.49$

$\mathrm{n}_{\text {Water }}=1.33$

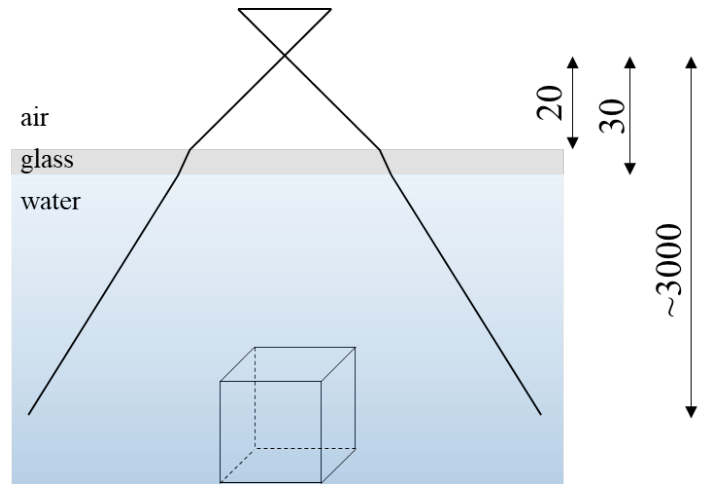

Figure 3. Configuration of the interfaces. A plane interface $(10 \mathrm{~mm}$ in thickness) of glass is simulated $20 \mathrm{~mm}$ in front of the principal point. The average distance to the object points is approx. $3 \mathrm{~m}$.

The simulated error-free image coordinates as well as corresponding object points are integrated into the bundle adjustment running standard software (AICON 3D Studio). Due to software technological restrictions, a non-relevant white noise of $10^{-6} \mathrm{~mm}$ is added to the error-free image coordinates. These coordinates are named error-free coordinates in the following. The result of the bundle adjustment contains interior orientation, exterior orientation and, in case of stereo cameras, the relative orientation parameters. The object points are introduced as fixed reference points.

For the following sections a notation will be used which describes the experiments in a structure as follows:

Number of Cameras Single/Multimedia Convergence-Ratio_ir/Water The example $2 \mathrm{Mm} 10^{\circ}-5 / 95$ describes an experiment with two cameras in a multimedia case (air-glass-water) with a convergence of $10^{\circ}$ equipped with a glass interface at $5 \%$ of the distance for each camera (bundle-invariant case) to the object. In case of single camera setting, of course, no convergence is part of the experiments naming.

\subsection{Single camera bundle adjustment}

To analyse the exterior and interior orientations within a bundle adjustment, two datasets, based on the setup of Figure 2, are simulated. For the first dataset $\left(1_{\mathrm{SM}}-1 / 99\right)$ perfect image coordinates without any refractive effects are used for the bundle adjustment, whereas the second dataset $\left(1_{\mathrm{MM}}-1 / 99\right)$ contains error-free refracted image coordinates. The interface is simulated $20 \mathrm{~mm}$ in front of the lens, thus leading to an air/water depth ratio of $\sim 1 / 99$. The bundle adjustment was performed using standard software not introducing any interfaces as parameters and using the standard collinearity equations with the distortion model according to (Brown, 1971). The interior and exterior orientations are determined within the bundle adjustment employing the object points as fixed reference points. Table 1 shows that the principal distance is longer in multimedia case by factor 1.42 . Furthermore, the values of the radial distortion parameters increase significantly, while other distortion parameters are calculated to zero, thus resembling the nominal values. According to (Lavest et al., 2003) the principal distance $\mathrm{c}$ increases underwater by a factor equivalent to the refraction index of water. (Taufiqur Rahman, 2013) presents ratios of 1.335 to 1.345 in experimental setups within identical environmental conditions and shows that the principal distance as well as the exterior orientations of a stereo camera system show high discrepancies between in-air and in-water calibration. (Agrafiotis and Georgopoulos, 2015) suggest not to overcome the refraction effects by camera calibration and show how the principal distance behaves in calibration depending on the ratio of air and water within the path of light.

\begin{tabular}{|l|r|r|r|r|}
\hline Experiment & $\begin{array}{c}\text { Principal } \\
\text { distance c } \\
{[\mathbf{m m}]}\end{array}$ & \multicolumn{1}{c|}{ A1 } & \multicolumn{1}{c|}{$\mathbf{A 2}$} & \multicolumn{1}{c|}{$\mathbf{A 3}$} \\
\hline nominal & 23.908 & 0.0 & 0.0 & 0.0 \\
\hline $1 \mathrm{sM}-1 / 99$ & 23.908 & $-1.5 \mathrm{e}-9$ & $4.2 \mathrm{e}-12$ & $-8.9 \mathrm{e}-15$ \\
\hline $1_{\mathrm{MM}-1 / 99}$ & 33.979 & $3.1 \mathrm{e}-4$ & $1.9 \mathrm{e}-7$ & $1.3 \mathrm{e}-10$ \\
\hline
\end{tabular}

Table 1. Interior orientation parameters (principal distance and radial distortion) of one single-medium dataset and one multimedia dataset.

Due to high correlations between c, A-parameters and $Z_{0}$ it might occur that the principal distance, radial distortion parameters and/or the exterior orientation will be calculated incorrectly. In contrast to the analysis of real datasets, analysing synthetic data allows for the comparison of the exterior orientations among different datasets. Figure 4 shows the systematic translation of exterior orientations between singlemedium and multimedia bundle along the optical axes of the respective camera. 


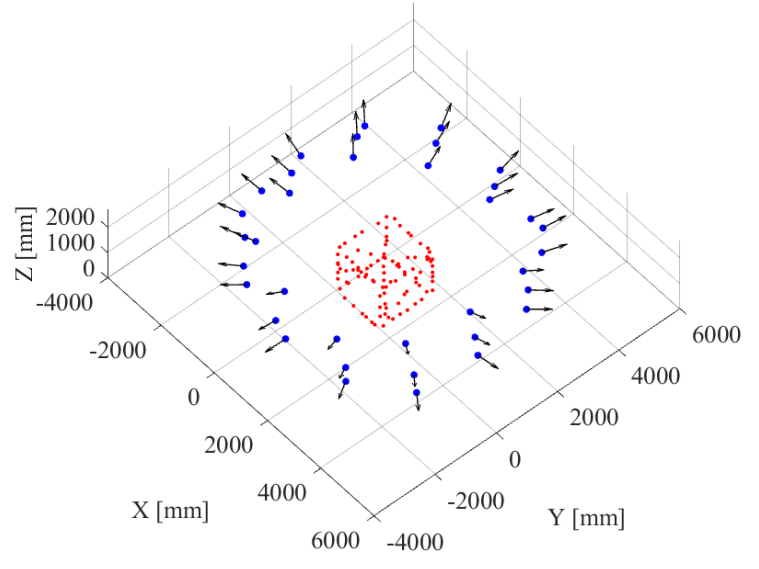

Figure 4. Exterior Orientations of $1 \mathrm{sM}^{-1 / 99}$ (blue dots). The vectors indicate the systematic difference between the exterior orientations of $1 \mathrm{sm}-1 / 99$ and $1 \mathrm{MM}-1 / 99$ of about $6.5 \mathrm{~mm}$ in average.

As long as the calculated orientation parameters are used within the bundle and object points are determined all in one process, the errors in object space might be irrelevant. The shifted exterior orientations in combination with the extended principal distance and calibrated distortion parameters represent the best mathematical solution for the multimedia case when using the collinearity equations.

The fact that the calibration in multimedia case can introduce an error to the interior (mainly the principal distance) and exterior orientation becomes more important when scale is provided not by reference points but by a stereo base.

\subsection{Stereo camera bundle adjustment}

Based on the setup with 36 images shown in Figure 2, 36 additional images are simulated. The additional images are calculated by adding a defined stereo base to the 36 main images. Accordingly, 36 image pairs are simulated, whereat each single camera has a dedicated interface (see Figure 5).

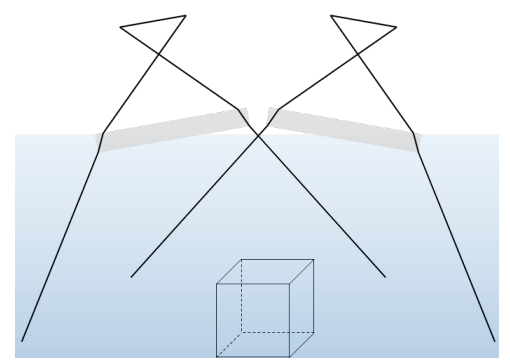

Figure 5. Convergent stereo camera setup with bundle-invariant interfaces.

The simulation of image coordinates for 36 image pairs is done the same way as explained previously with the interface $20 \mathrm{~mm}$ in front of the principal point, a base of $200 \mathrm{~mm}$ and no convergence, leading to the following datasets.

$2 \mathrm{SM} 0^{\circ}-1 / 99$

$2 \mathrm{MM} 0^{\circ}-1 / 99$

When analysing these datasets, the same effects as in section 3.1 can be observed. The principal distance becomes larger and the radial distortion parameters differ significantly from the nominal parameters in multimedia case. In addition, the exterior orientations of the multimedia dataset show systematic translations in direction of the optical axes as in the singlecamera case illustrated in Figure 4. When scale is provided by the relative orientation, it is essential that the relevant parameters are adjusted correctly. The following section discusses the behaviour of the relative orientation when using standard software and points out the critical aspect when scale is provided by the relative orientation only.

3.2.1 Variation of convergence: The determinability of the relative orientation depends on the stereo configuration. The results in Table 2 show different datasets, whereat the angle of convergence varies by $5^{\circ}$ at each dataset from $0^{\circ}$ to $25^{\circ}$.

\begin{tabular}{|c|c|c|c|c|c|c|c|c|}
\hline \multirow[b]{2}{*}{ No. } & \multirow[b]{2}{*}{ Dataset } & \multicolumn{6}{|c|}{ Relative orientation } & \multirow[b]{2}{*}{$\begin{array}{c}c \\
{[\mathbf{m m}]}\end{array}$} \\
\hline & & $\begin{array}{c}\mathbf{X}_{\mathbf{0}} \\
{[\mathbf{m m}]}\end{array}$ & $\left.\begin{array}{c}\mathbf{Y}_{0} \\
{[\mathbf{m m}]}\end{array}\right]$ & $\left.\begin{array}{c}\mathbf{Z}_{0} \\
{[\mathrm{~mm}]}\end{array}\right]$ & $\begin{array}{c}\omega \\
{\left[^{\circ}\right]}\end{array}$ & $\begin{array}{c}\varphi \\
\left.{ }^{\circ}\right]\end{array}$ & $\begin{array}{c}\boldsymbol{\kappa} \\
{\left[{ }^{\circ}\right]}\end{array}$ & \\
\hline 0 & nominal & 200.000 & 0.000 & 0.000 & 0.000 & $0-25$ & 0.000 & 23.908 \\
\hline 1 & $2_{\mathrm{SM}^{-}} 0^{\circ}-1 / 99$ & 00.000 & 0.000 & 0.000 & 0.000 & 0.000 & 0.000 & 23.908 \\
\hline 2 & $2 \mathrm{MM}^{-} 0^{\circ}-1 / 99$ & 199.994 & 0.000 & 0.019 & 0.000 & 0.000 & 0.000 & 33.979 \\
\hline 3 & $2 \mathrm{MM}^{-} 5^{\circ}-1 / 99$ & 00.572 & 0.000 & -0.062 & 0.000 & 5.001 & 0.000 & 33.978 \\
\hline 4 & $2 \mathrm{MM}^{-1}-10^{\circ}-1 / 99$ & 01.143 & -0.002 & -0.116 & 0.000 & 10.000 & 0.000 & 33.978 \\
\hline 5 & $2 \mathrm{MM}^{-1}-15^{\circ}-1 / 99$ & 201.715 & -0.007 & -0.192 & 0.000 & 15.003 & 0.000 & 33.97 \\
\hline 6 & $22_{\mathrm{MM}}-20^{\circ}-1 / 99$ & 202.266 & -0.010 & -0.339 & -0.001 & 20.003 & 0.000 & 33.979 \\
\hline 7 & $22_{\mathrm{MM}}-25^{\circ}-1 / 99$ & 202.884 & -0.011 & -0.378 & 0.001 & 25.004 & 0.000 & 33.98 \\
\hline
\end{tabular}

Table 2. Relative orientation and principal distance as a result of the bundle adjustment running standard software for different datasets with varying angle of convergence.

The dataset 1 confirms that the mathematical model is correct for the single-medium case, since all parameters of the relative orientation equal the nominal parameters. As already noted, the camera positions of all multimedia datasets are systematically estimated further away from the object. Dataset 2 shows slightly different results for $\mathrm{X}_{0}$ and $\mathrm{Z}_{0}$ (direction of the optical axis) of the relative orientation and the expected longer principal distance. This parallel dataset suggests a nearly correct estimation of the relative orientation, thus leading to the adoption that scale might be correct. The convergent datasets show a significant change in relative orientation, whereby the most convergent configuration leads to the most effected orientation parameters. Although the variation of the relative orientation already indicates an incorrect modelling of refractive effects, a statement to the quality of the datasets can only be given by analysing calculated object points. Since object coordinates will always result from jointly processing interior, relative and exterior orientation, it is necessary to take all of these parameters into account to evaluate the quality of the calibration.

To evaluate the actual error in object space, 3D coordinates are calculated. The error-free image coordinates are introduced to a bundle adjustment using all 36 synthetic image pairs to determine the relative (s. Table 2), exterior and interior orientation parameters. The relative orientation is introduced as a constraint and adjusted. To calculate 3D points, the calibrated parameters of exterior and interior orientation as well as the error-free image coordinates of one of the 36 image pairs are used in forward intersection. Since the relative orientation is introduced as a constraint, it is responsible for the scaling. The 
intersected points can then be compared with the known reference object points. The $3 \mathrm{D}$ error vectors of the object points calculated from one image pair are shown exemplary in Figure 6.

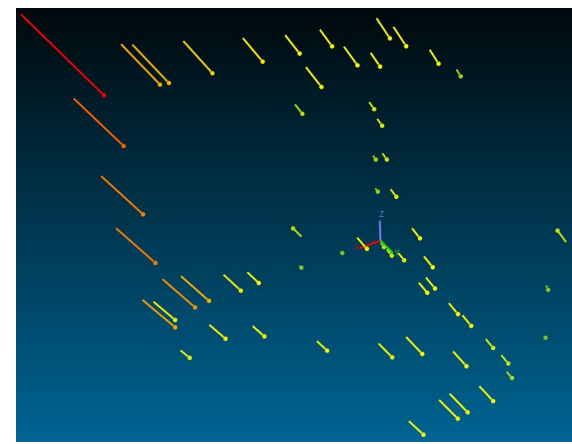

\begin{tabular}{|r|r|r|}
\hline $\begin{array}{c}\boldsymbol{\varphi} \\
{\left[{ }^{\circ}\right]}\end{array}$ & $\begin{array}{c}\text { Mean } \\
{[\mathbf{m m}]}\end{array}$ & $\begin{array}{c}\text { Max } \\
{[\mathbf{m m}]}\end{array}$ \\
\hline 0 & 0.25 & 1.74 \\
\hline 5 & 0.20 & 1.13 \\
\hline 10 & 0.54 & 2.70 \\
\hline 15 & 0.80 & 3.43 \\
\hline 20 & 0.95 & 4.33 \\
\hline 25 & 1.16 & 4.97 \\
\hline
\end{tabular}

Figure 6. 3D error vectors (exaggerated by factor 230) of forward intersected 3D points of one exemplary image pair (dataset $2 \mathrm{MM}-20^{\circ}-1 / 99$ ) using the calibrated parameters of interior and relative orientation. The mean and max values of the $3 \mathrm{D}$ errors are depicted in the table for each convergent dataset $\left(\varphi=0^{\circ}\right.$ to $\left.25^{\circ}\right)$.

Since the 3D points are calculated using the adjusted exterior orientations, they exist in the same coordinate system as the reference and do not have to be fitted to these. Figure 6 visualises the systematic deviations. Besides the absolute translation in the direction of observation axis of the particular image pair, an error in scale can be observed in forward intersected points. While the dataset of parallel cameras shows small errors, the errors become larger with increasing convergence. Due to the correlated parameters the implicit form does not model the system correctly. The calibrated parameters of interior and relative orientation are not valid for the whole object space. This becomes critical when the object is not flat, which is the case in most applications.

The relative orientation is almost calibrated correctly for the parallel dataset (s. Table 2, dataset 2). When users do not have any chance to verify the relative and interior orientation parameters, the calibration results might suggest good quality. However, the analysis of the synthetic data points out, that due to correlation of exterior, interior and relative orientation the calculated $3 \mathrm{D}$ points can be incorrect. Thus, the strategy to use standard software for calibrating the interior and the relative orientation in-situ underwater is not suitable to overcome refractive effects according to this synthetic data. When calibration is done with imperfect image coordinates (real images) using a flat calibration target, these relations could become even more critical, since the correlations between critical parameters are higher in general.

3.2.2 Variation of air/water ratio: The refractive effects depend, apart from the refractive indices, on the distances the ray of light travels through each medium. In many applications a camera is mounted in a watertight housing, thus the interface is placed nearly perpendicular right in front of the camera lens. In close-range applications the ratio of distance through air and water can become close to $1 / 1$ whereas in typical ROV applications the ratio becomes smaller (e.g. 1/300).

In this section, the ratio of the distance the ray of light travelled through air and water is varied to analyse the effects on the orientation parameters and the effects in object space. Only the parallel configuration $2 \mathrm{MM}-0^{\circ}-\mathrm{X} / \mathrm{X}$ is used for the tests, since the convergent datasets showed unacceptable results for the relative orientation (see section 3.2.1) when using standard software and modelling refractive effects implicitly. The implicit modelling with varied air/water ratio for convergent configurations should be worse than for parallel setups.

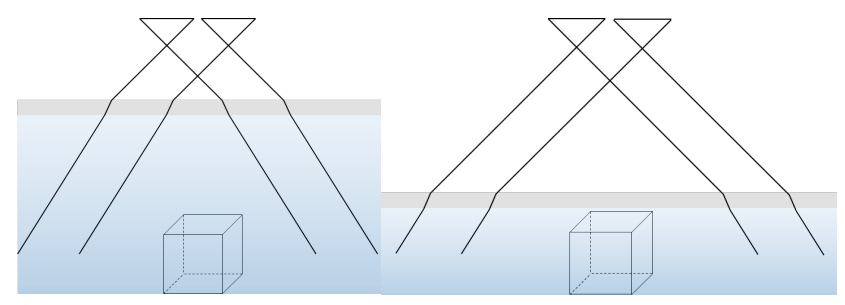

Figure 7. Illustration of the refractive effects with different air to water ratio.

Table 3 shows the result for the relative orientation parameters and the principal distance estimated by the bundle adjustment with the dataset $2 \mathrm{Mm}^{-} 0^{\circ}-\mathrm{X} / \mathrm{X}$ being shifted away from the camera by $10 \%$ successively. The dataset number 1 represents the case of a typical underwater housing with an air/water depth ratio of $1 / 99$. This dataset shows only a small difference in the stereo base. When moving the interface away from the camera, the error of the relative orientation raises up to more than $2 \mathrm{~mm}$ $\left(2 \mathrm{MM}-0^{\circ}-50 / 50\right)$. The results are not stable, not even with synthetic data. They highly depend on the configuration of the bundle and the correlations between critical parameters. As expected, the principal distance becomes smaller, when the percentage of water is decreased. As presented by (Agrafiotis and Georgopoulos, 2015), using standard software might lead to contrariwise results regarding the principal distance. In contrast to the experiments of the previous section, the variation of the interface leads to large errors of the relative orientation in $Z_{0}$, which represents the direction of the optical axis of the stereo camera.

\begin{tabular}{|c|c|c|c|c|c|c|c|c|}
\hline \multirow[b]{2}{*}{ No. } & \multirow[b]{2}{*}{ Dataset } & \multicolumn{6}{|c|}{ Relative orientation } & \multirow[b]{2}{*}{$\begin{array}{c}\mathbf{c} \\
{[\mathbf{m m}]}\end{array}$} \\
\hline & & $\begin{array}{c}\mathbf{X}_{0} \\
{[\mathrm{~mm}]}\end{array}$ & \begin{tabular}{|c|}
$\mathbf{Y}_{\mathbf{0}}$ \\
{$[\mathbf{m m}]$}
\end{tabular} & \begin{tabular}{|c|}
$\mathrm{Z}_{0}$ \\
{$[\mathrm{~mm}]$}
\end{tabular} & $\begin{array}{l}\omega \\
{\left[{ }^{\circ}\right]}\end{array}$ & $\begin{array}{c}\varphi \\
{\left[{ }^{\circ}\right]}\end{array}$ & $\begin{array}{c}\mathbf{K} \\
{\left[{ }^{\circ}\right]}\end{array}$ & \\
\hline 0 & nomi & 0.000 & 0.000 & 0.000 & 0.000 & $0-25$ & 0.000 & 23.908 \\
\hline 1 & $\mathrm{M}-0^{\circ}-1 /$ & 9.994 & 0.000 & 0.019 & 0.000 & 0.000 & 0.000 & $33.9^{\prime}$ \\
\hline 2 & $2 \mathrm{MM}-0^{\circ}-10 / 90$ & .912 & -0.018 & 0.012 & -0.001 & -0.008 & 0.001 & 33.8 \\
\hline 3 & $2 \mathrm{MM}^{-} 0^{\circ}-20 / 80$ & 0 & 0.021 & 1.491 & 0.003 & 0.018 & 0.001 & 33.8 \\
\hline 4 & $2 \mathrm{MM}^{-\mathrm{C}}$ & 9.732 & 0.142 & 1.501 & 0.019 & 0.002 & -0.002 & 33.744 \\
\hline 5 & $\mathrm{LMM}^{-\mathrm{O}^{\mathrm{T}}}$ & 9.664 & 0.219 & 1.594 & 0.018 & 0.015 & -0.001 & 33.654 \\
\hline 6 & 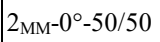 & 99.702 & 0.234 & 2.345 & 0.021 & 0.033 & 0.0 & 33.549 \\
\hline
\end{tabular}

Table 3. Relative orientation and principal distance as a result of the bundle adjustment using standard software for different datasets with varying air to water ratio.

To quantify the effect of the incorrectly determined relative orientation, forward intersections with calibrated orientation parameters are performed following the same procedure as explained in the previous section. The 3D errors of intersected points are visualised for an exemplary image pair in Figure 8. 


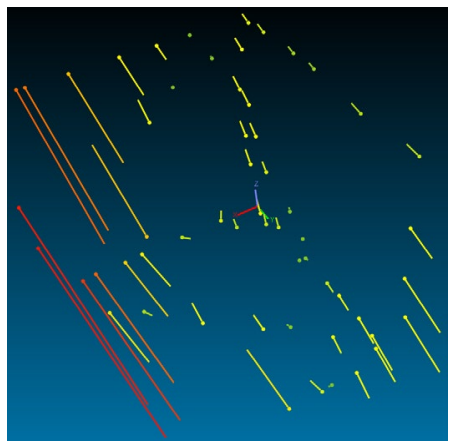

\begin{tabular}{|c|r|r|}
\hline R & $\begin{array}{r}\text { Mean } \\
\text { [mm] }\end{array}$ & $\begin{array}{c}\text { Max } \\
\text { [mm] }\end{array}$ \\
\hline $1 / 99$ & 0.25 & 1.74 \\
\hline $10 / 90$ & 4.06 & 28.47 \\
\hline $20 / 80$ & 6.45 & 55.08 \\
\hline $30 / 70$ & 12.37 & 107.48 \\
\hline $40 / 60$ & 17.77 & 79.13 \\
\hline $50 / 50$ & 93.42 & 261.47 \\
\hline
\end{tabular}

Figure 8. 3D error vectors (exaggerated by factor 100) of forward intersected 3D points of one exemplary image pair (dataset $2 \mathrm{MM}-0^{\circ}-10 / 90$ ) using the calibrated parameters of interior and relative orientation. The mean and max values of the $3 \mathrm{D}$ errors are depicted in the table for each air/water ratio.

The calibrated orientation parameters differ from the nominal values in a similar manner as the calibrated values of the convergent datasets shown in section 3.2.1. However, when analysing the error in object space, it becomes clear that the implicit form cannot model the system correctly. The larger the depth ratio of different media becomes, the more critical the errors in object space seem to be. Meaning that in close-range applications where the ratio becomes large (e.g. 1/1), modelling the refractive effects implicitly will lead to gross errors, whereas for applications with smaller ratios (e.g. 1/300) the modelling errors are smaller.

Depending on the application and the requirements for quality, using standard software and an implicit modelling might be sufficient. The synthetic datasets for a perfectly aligned parallel setup, an air/water ratio of 1/99 and a favourable geometrical bundle configuration lead to $\sim 0.25 \mathrm{~mm}$ error in object space, which corresponds to an accuracy of $1 / 20$ pixel in image space. However, when using real data and poor bundle configurations, it is not clear how big errors in object space are. Furthermore, the errors can become large easily when the correlations are critical and the setup is not aligned perfectly.

\section{ANALYSIS OF CALIBRATION AND ORIENTATION FOR PLANAR INTERFACES IN EXPLICIT FORM}

As stated in the previous section, the implicit modelling of refractive interfaces is critical due to highly correlated parameters. In this section, the synthetic data from section 3 will be analysed using a strict explicit model. It is assumed that due to the strict model, the synthetic data will not lead to systematic errors in object space as observed when running standard software using implicit modelling.

\subsection{Explicit modelling}

The use of standard software and the implicit modelling of refractive effects can lead to relevant errors in object space. Therefore a multimedia bundle adjustment explicitly modelling the refractive interfaces according to the publication of (Mulsow, 2010) was implemented. In our implementation, the interior orientation is assumed to be known and fixed for the following analysis. In real tasks, the interior and the relative orientation could be determined by standard calibration procedure in air using existing software products (see case 4 and 5, Figure 1). As discussed in section 2.3, the case 4 of Figure 1 is marginally realistic due to instability of the mechanical system. The simultaneous determination of the parameters of interfaces, interior and relative orientation (see case 3 , Figure 1) is theoretically possible, but will probably lead to a very unstable numerical system.

To be able to calculate a relative orientation in multimedia case, some parameters are introduced to the bundle adjustment:

- $\mathrm{n}_{\text {air }}, \mathrm{n}_{\text {glass }}, \mathrm{n}_{\text {water }}, \mathrm{N} 1_{\mathrm{x}}, \mathrm{N} 1_{\mathrm{y}}, \mathrm{N} 1_{\mathrm{z}}, \mathrm{d} 1, \mathrm{~N} 2_{\mathrm{x}}, \mathrm{N} 2_{\mathrm{y}}, \mathrm{N} 2_{\mathrm{z}}, \mathrm{d} 2$, $\mathrm{X}_{0}, \mathrm{Y}_{0}, \mathrm{Z}_{0}, \omega, \varphi, \kappa$

\begin{tabular}{|c|c|c|}
\hline \multirow[t]{7}{*}{ where } & $\mathrm{n}_{\text {air }}$ & $=$ refractive index of air \\
\hline & nglass & $=$ refractive index of glass \\
\hline & $\mathrm{n}_{\text {water }}$ & $=$ refractive index of water \\
\hline & $\mathrm{N} 1_{\mathrm{x}}, \mathrm{N} 1_{\mathrm{y}}, \mathrm{l}$ & $1=$ plane parameters of interface 1 \\
\hline & $\mathrm{N} 2 \mathrm{x}, \mathrm{N} 2 \mathrm{y}, \mathrm{l}$ & $2=$ plane parameters of interface 2 \\
\hline & $\mathrm{X}_{0}, \mathrm{Y}_{0}, \mathrm{Z}_{0}$ & $=$ translation of relative orientation \\
\hline & $\omega, \varphi, \kappa$ & $=$ rotation of relative orientation \\
\hline
\end{tabular}

The first interface represents the plane of refraction from medium air to glass, the second one the plane of refraction from glass to water. The thickness of the interfaces as well as the refractive indices of air and glass are assumed to be known, whereas the other added parameters are treated as unknowns.

\subsection{Synthetic data}

First of all, the datasets from Table 2 and Table 3 were analysed again using the multimedia bundle adjusting the exterior orientations and the relative orientation. The results of the adjustment equal the nominal synthetic values, which are introduced into the bundle. Furthermore, a combination of the most critical datasets $\left(25^{\circ}\right.$ convergence and air/water ratio of $50 / 50)$ is adjusted via the multimedia bundle. Even this critical configuration converges correctly, thus proving the implementation of the multimedia bundle adjustment.

\begin{tabular}{|c|l|c|c|c|c|c|c|}
\hline \multirow{2}{*}{ No. } & \multirow{2}{*}{ Dataset } & \multicolumn{6}{|c|}{ Relative orientation } \\
\cline { 2 - 8 } & $\begin{array}{c}\mathbf{X}_{\mathbf{0}} \\
{[\mathbf{m m}]}\end{array}$ & $\begin{array}{c}\mathbf{Y}_{\mathbf{0}} \\
{[\mathbf{m m}]}\end{array}$ & $\begin{array}{c}\mathbf{Z}_{\mathbf{0}} \\
{[\mathbf{m m}]}\end{array}$ & $\begin{array}{c}\boldsymbol{\omega} \\
{\left[{ }^{\circ}\right]}\end{array}$ & $\begin{array}{c}\boldsymbol{\varphi} \\
{\left[{ }^{\circ}\right]}\end{array}$ & $\begin{array}{c}\mathbf{K} \\
{\left[{ }^{\circ}\right]}\end{array}$ \\
\hline 0 & nominal & 200.000 & 0.000 & 0.000 & 0.000 & $0-25$ & 0.000 \\
\hline 1 & $2_{\mathrm{MM}}-25^{\circ}-1 / 99$ & 200.000 & 0.000 & 0.000 & 0.000 & 25.000 & 0.000 \\
\hline 2 & $22_{\mathrm{MM}}-0^{\circ}-50 / 50$ & 200.000 & 0.000 & 0.000 & 0.000 & 0.000 & 0.000 \\
\hline 3 & $2 \mathrm{MM}^{\mathrm{M}}-25^{\circ}-50 / 50$ & 200.000 & 0.000 & 0.000 & 0.000 & 25.000 & 0.000 \\
\hline
\end{tabular}

Table 4. Relative orientation as a result of the multimedia bundle adjustment for the most critical datasets.

To prove robustness of the process, realistic noise for observations as well as for first approximations for unknown values is introduced as follows:

$\begin{array}{llrr}- & \mathrm{n}_{\text {water }} & \pm & 0.01 \\ - & \text { Image coordinates } & \pm & 1 \mathrm{pixel} \\ \text { - } & \text { Translation of exterior orientations } & \pm & 200 \mathrm{~mm} \\ \text { - } & \text { Rotation of exterior orientations } & \pm & 1^{\circ}\end{array}$

The results converge to the same values as without noise, which verifies our implementation with respect to the modelled parameters. Furthermore, the results of forward intersected points do not show any significant errors in object space as expected with simulated data. 


\section{REAL DATA EXPERIMENT}

To prove the findings of the analysis of synthetic data and to quantify an error, a real experiment is conducted. Figure 9 shows the setup of the experiment. Two single cameras in waterproof housings are arranged having an angle of convergence of $\sim 23^{\circ}$. The cameras capture images of a $3 \mathrm{D}$ test field from about $300 \mathrm{~mm}$ distance in average.

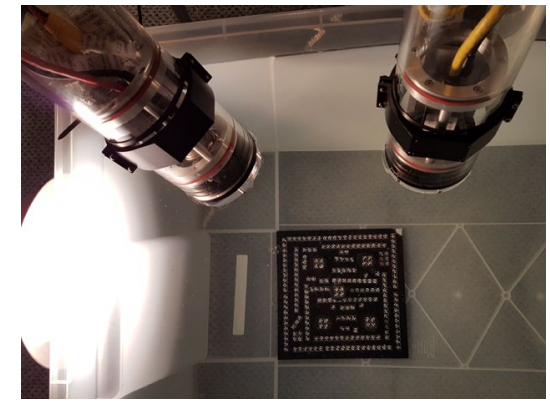

Figure 9. Convergent underwater stereo camera observing a test field underwater.

First, the interior orientation of each camera is calibrated in air using standard software. After mounting the acryl glass interface, the cameras are set up under water (s. Figure 9). To calculate the relative orientation, two methods are used. Firstly, standard software is used adjusting the interior, exterior and relative orientation parameters. Secondly, our own implementation is used to determine the exterior and relative orientation parameters as well as additional parameters such as the refractive index of water. The interior orientation is kept fixed by pre calibration.

Table 5 shows the intrinsic camera parameters for the left camera exemplary (right camera is similar) of the pre calibration in air and of the calibration under water using standard software. The principal distance raises by factor 1.51 underwater. Furthermore, all distortion parameters (A, B, C) change significantly from air to water bundle adjustment. In the multimedia bundle adjustment, $\mathrm{C} 1$ cannot be determined significantly, whereas in air, all parameters are adjusted significantly.

\begin{tabular}{|c|c|c|c|c|c|c|c|c|c|c|}
\hline \multirow{2}{*}{ Data } & $\begin{array}{c}\mathbf{c} \\
{[\mathbf{m m}]}\end{array}$ & $\begin{array}{c}\mathbf{x h} \\
{[\mathbf{m m}]}\end{array}$ & $\begin{array}{c}\text { yh } \\
{[\mathbf{m m}]}\end{array}$ & $\mathbf{A 1}$ & $\mathbf{A 2}$ & $\mathbf{A 3}$ & $\mathbf{B 1}$ & B2 & C1 & C2 \\
\cline { 2 - 10 } & $\mathbf{S c}$ & $\mathbf{S x h}$ & $\mathbf{S y h}$ & SA1 & SA2 & SA3 & SB1 & SB2 & SC1 & SC2 \\
\hline \multirow{2}{*}{$\begin{array}{c}\text { IOR } \\
\text { AIR }\end{array}$} & 4.367 & 0.145 & 0.005 & $-1.5 \mathrm{e}-4$ & $3.6 \mathrm{e}-4$ & $-4.8 \mathrm{e}-6$ & $3.1 \mathrm{e}-5$ & $1.3 \mathrm{e}-5$ & $8.7 \mathrm{e}-5$ & $-5.0 \mathrm{e}-5$ \\
\cline { 2 - 10 } & $3.9 \mathrm{e}-4$ & $4.3 \mathrm{e}-4$ & $4.0 \mathrm{e}-4$ & $6.6 \mathrm{e}-6$ & $5.4 \mathrm{e}-7$ & $1.5 \mathrm{e}-8$ & $1.2 \mathrm{e}-6$ & $1.3 \mathrm{e}-6$ & $1.3 \mathrm{e}-5$ & $1.3 \mathrm{e}-5$ \\
\hline $\begin{array}{c}\text { IOR } \\
\text { Water }\end{array}$ & 6.595 & 0.128 & -0.016 & $-2.6 \mathrm{e}-3$ & $9.0 \mathrm{e}-5$ & $6.0 \mathrm{e}-8$ & $3.8 \mathrm{e}-4$ & $-6.0 \mathrm{e}-4$ & $-8.6 \mathrm{e}-6$ & $-2.1 \mathrm{e}-4$ \\
\cline { 2 - 8 } & $4.3 \mathrm{e}-4$ & $4.7 \mathrm{e}-4$ & $4.5 \mathrm{e}-4$ & $7.0 \mathrm{e}-6$ & $7.2 \mathrm{e}-7$ & $2.3 \mathrm{e}-8$ & $3.1 \mathrm{e}-6$ & $2.7 \mathrm{e}-6$ & $1.3 \mathrm{e}-5$ & $1.3 \mathrm{e}-5$ \\
\hline
\end{tabular}

Table 5. Interior orientation parameters and their standard deviation of left camera of bundle adjustment using standard software in air and in underwater configuration.

The relative orientation of the calibration in air is not determined, since it would not be comparable to the one determined underwater. When placing the interface in front of the camera, the relative configuration of the camera changes due to necessarily touching and moving of the camera housings. However, the relative orientation of the two approaches are compared in the following table.

\begin{tabular}{|c|c|c|c|c|c|c|}
\hline \multirow{2}{*}{ Dataset } & \multicolumn{5}{|c|}{ Relative orientation } \\
\cline { 2 - 7 } & $\begin{array}{c}\mathbf{X}_{\mathbf{0}} \\
{[\mathbf{m m}]}\end{array}$ & $\begin{array}{c}\mathbf{Y}_{\mathbf{0}} \\
{[\mathbf{m m}]}\end{array}$ & $\begin{array}{c}\mathbf{Z}_{\mathbf{0}} \\
{[\mathbf{m m}]}\end{array}$ & $\begin{array}{c}\boldsymbol{\omega} \\
{\left[{ }^{\circ}\right]}\end{array}$ & $\begin{array}{c}\boldsymbol{\varphi} \\
{\left[{ }^{\circ}\right]}\end{array}$ & $\begin{array}{c}\mathbf{K} \\
{\left[{ }^{\circ}\right]}\end{array}$ \\
\hline implicit & 216.130 & 62.475 & 3.374 & 0.826 & 22.529 & 58.804 \\
\hline explicit & 215.076 & 62.437 & 7.873 & 0.462 & 22.669 & 58.840 \\
\hline
\end{tabular}

Table 6. Relative orientation of underwater bundle adjustment using standard software (implicit modelling) and own implementation (explicit modelling).

It can be seen that the relative orientation of the two approaches differs significantly. To quantify an error in object space, it is inevitable to calculated 3D points. Since different adjusted interior and exterior orientations form a mathematical model with the adjusted relative orientation, the whole system needs to be analysed. As done for synthetic data, forward intersection is used to compute 3D points from one image pair based on the adjusted orientations.

When comparing the 3D points calculated from an image pair captured at an average distance to the calibration object, no significant differences between the two approaches can be detected. When comparing the 3D points calculated from an image pair closer to the camera, a significant difference between the two approaches can be identified. While the mean error of the implicit modelling increases, the error of the explicit form is similar to the error of the other image pair.

\begin{tabular}{|c|c|r|r|}
\hline $\begin{array}{c}\text { Image } \\
\text { pair }\end{array}$ & Model & $\begin{array}{c}\text { Mean } \\
{[\mathbf{m m}]}\end{array}$ & \multicolumn{1}{c|}{$\begin{array}{c}\text { Max } \\
{[\mathbf{m m}]}\end{array}$} \\
\hline \multirow{2}{*}{$\begin{array}{c}\text { Average } \\
\text { distance }\end{array}$} & implicit & 0.081 & 0.227 \\
\cline { 2 - 4 } & explicit & 0.072 & 0.254 \\
\hline \multirow{2}{*}{$\begin{array}{c}\text { Close to } \\
\text { camera }\end{array}$} & implicit & 0.132 & 0.638 \\
\cline { 2 - 4 } & explicit & 0.067 & 0.409 \\
\hline
\end{tabular}

Table 7. 3D error of 3D points calculated from two image pairs.

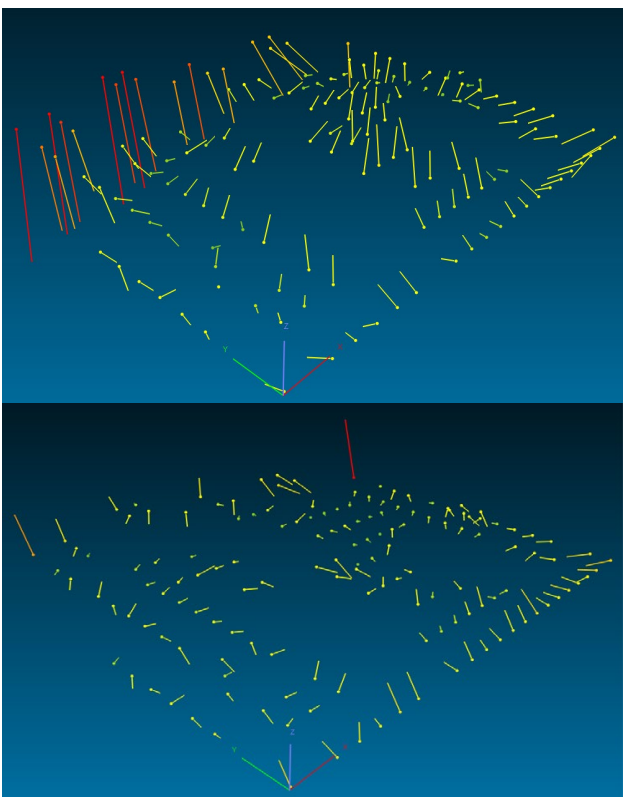

Figure 10. 3D error vectors (exaggerated by factor 75 ) of the $3 \mathrm{D}$ points calculated from the closer image pair for implicit form (top) and explicit form (bottom). 
It seems that due to the incorrect modelling, the calibrated parameters of the bundle adjustment of the implicit form is not valid for the whole object space. Since the air/water ratio is small in the experiment, the implicit form does model the system almost as good as our implementation with strict modelling of the interfaces. Nevertheless, the strict model shows its advantages when calculating points far from the average calibration distance proving the implicit model is not strictly correct. It is noticeable that it might occur that the errors of implicit modelling, depending on the calibration technique, the object to be measured and the setup of the multi-camera system are overlaid by poor bundle configuration effects and poor underwater image quality effects.

\section{CONCLUSION}

This contribution refers to different articles of underwater photogrammetry and computer vision. An overview of system configurations and calibration techniques is given and the investigation of the behaviour of relative orientation and interior orientation of a multi-camera system with planar interfaces within the bundle adjustment is covered. Synthetic datasets prove theoretical considerations and show how implicit calibration might affect the $3 \mathrm{D}$ reconstruction. It can be seen that the error in object space highly depends on the setup of cameras and interfaces. Furthermore, the configuration of the bundle and of the reference object for calibration affect the dimension of introduced errors. The correlations between the principal distance and the camera positions become critical when the scale is provided by the relative orientation of multicamera systems. Systematic errors in absolute location and geometric deformation of forward intersected points can be detected in simulated datasets as well as in a real dataset. Even if for some cases standard collinearity equations might model the system quite well, it is highly recommended not to neglect refractive interfaces. By modelling these, the quality for $3 \mathrm{D}$ points can be improved significantly.

\section{ACKNOWLEDGEMENT}

This work is based on three projects, funded by the European Regional Development Fund (EFRE, ZW 6-85003503 and 6-85007826) and by the Volkswagen Foundation, Germany (ZN3253).

\section{REFERENCES}

Agrafiotis, P., Georgopoulos, A., 2015. Camera constant in the case of two media photogrammetry. ISPRS - International Archives of the Photogrammetry, Remote Sensing and Spatial Information Sciences XL-5/W5, 1-6.

Bräuer-Burchardt, C., Heinze, M., Schmidt, I., Kühmstedt, P., Notni, G., 2015. Compact handheld fringe projection based underwater 3D-scanner. ISPRS - International Archives of the Photogrammetry, Remote Sensing and Spatial Information Sciences XL-5/W5, 33-39.

Brown, D.C., 1971. Close-Range Camera Calibration. In: Photogrammetric Engineering, pp. 855-866.

Drap, P., Seinturier, J., Hijazi, B., Merad, D., Boi, J.-M., Chemisky, B., Seguin, E., Long, L., 2015. The ROV 3D Project. Journal on Computing and Cultural Heritage 8 (4), 124.
Kotowski, R., 1987. Zur Berücksichtigung lichtbrechender Flächen im Strahlenbündel. Zugl.: Bonn, Univ., Diss., 1986. Beck, München.

Lavest, J.M., Rives, G., Laprest, J.T., 2003. Dry camera calibration for underwater applications. Machine Vision and Applications 13 (5-6), 245-253.

Maas, H.-G., 1992. Diss. Digitale Photogrammetrie in der dreidimensionalen Strömungsmesstechnik. ETH Zürich Dissertation Nr. 9665.

Maas, H.-G., 2015. A modular geometric model for underwater photogrammetry. ISPRS - International Archives of the Photogrammetry, Remote Sensing and Spatial Information Sciences XL-5/W5, 139-141.

Menna, F., Nocerino, E., Fassi, F., Remondino, F., 2016. Geometric and Optic Characterization of a Hemispherical Dome Port for Underwater Photogrammetry. Sensors (Basel, Switzerland) 16 (1).

Menna, F., Nocerino, E., Remondino, F., 2017a. Flat versus hemispherical dome ports in underwater photogrammetry. ISPRS - International Archives of the Photogrammetry, Remote Sensing and Spatial Information Sciences XLII-2/W3, 481-487.

Menna, F., Nocerino, E., Remondino, F., 2017b. Optical aberrations in underwater photogrammetry with flat and hemispherical dome ports. In: Remondino, F., Shortis, M.R. (Eds.), SPIE Optical Metrology. SPIE, p. 1033205.

Menna, F., Nocerino, E., Troisi, S., Remondino, F., 2013. A photogrammetric approach to survey floating and semisubmerged objects. In: Remondino, F., Shortis, M.R., Beyerer, J., Puente León, F. (Eds.), SPIE Optical Metrology 2013. SPIE, $87910 \mathrm{H}$.

Mulsow, C., 2010. A flexible multi-media bundle approach. ISPRS - International Archives of the Photogrammetry, Remote Sensing and Spatial Information Sciences XXXVIII/5, 472477.

Nocerino, E., Menna, F., Fassi, F., Remondino, F., 2016. Underwater calibration of dome port pressure housings. ISPRS - International Archives of the Photogrammetry, Remote Sensing and Spatial Information Sciences XL-3/W4, 127-134.

Rofallski, R., Luhmann, T., 2018. Fusion von Sensoren mit optischer 3D-Messtechnik zur Positionierung von Unterwasserfahrzeugen. Hydrographie 2018. Trend zu unbemannten Messsystemen. DVW-Schriftenreihe, Band 91. Wißner-Verlag, Augsburg, pp. 223-234.

Shortis, M., 2015. Calibration Techniques for Accurate Measurements by Underwater Camera Systems. Sensors (Basel, Switzerland) 15 (12), 30810-30826.

Shortis, M., Harvey, E., Seager, J., 2007. A Review of the Status and Trends in Underwater Videometric Measurement.

Taufiqur Rahman, 2013. Oceans - San Diego, 2013. Conference; 23 - 27 Sept. 2013, San Diego, California. IEEE, Piscataway, NJ. 